\title{
Nanovesicle-mediated delivery of anticancer agents effectively induced cell death and regressed intrahepatic tumors in athymic mice
}

\author{
Joseph George $\mathbb{D}^{1,2,3} \cdot$ Irene K. Yan ${ }^{1,2} \cdot$ Tushar Patel $\mathbb{1}^{1,2}$
}

Received: 7 September 2017 / Revised: 27 January 2018 / Accepted: 12 February 2018 / Published online: 10 May 2018

(c) United States \& Canadian Academy of Pathology 2018

\begin{abstract}
Hepatocellular carcinoma is highly resistant to chemotherapy. Here we evaluated the use and efficacy of milk-derived nanovesicles (MNV) as an approach to improve delivery of anticancer agents into HCC cells and intrahepatic tumors. We developed a protocol for isolation of MNVs from skim milk using ultracentrifugation, and characterized using nanoparticle tracking analysis (NTA) and electron microscopy. MNVs were loaded with doxorubicin (dox-MNV) or miR221 antisense oligonucleotides (anti-miR221-MNV), and further evaluated using spectrophotometry, NTA, and zeta potential measurements. HepG2, Hep3B, and PLC/PRF/5 HCC cells in culture were treated with dox-MNV and anti-miR221MNV and evaluated with drug delivery and anticancer activity. The efficacy of dox-MNV and anti-miR221-MNV to arrest tumor growth in vivo was assessed on intrahepatic tumors induced in nude mice. Cellular uptake studies showed plain and dox-MNV attained saturation within $4 \mathrm{~h}$ of treatment. Cytotoxicity studies on HepG2, Hep3B, and PLC/PRF/5 HCC cells with dox-MNV at $1 \mu \mathrm{M}$ resulted in $20 \%$ cell death at $24 \mathrm{~h}, 50 \%$ at $48 \mathrm{~h}$, and $80 \%$ at $72 \mathrm{~h}$. HepG2 cells treated with doxMNV and anti-miR221-MNV exhibited nuclear disintegration, and apoptosis within $24 \mathrm{~h}$. Combination treatment of intrahepatic tumors with dox-MNV and anti-miR221-MNV resulted in marked reduction of tumor size and increased survival rate in nude mice. Our studies demonstrated that MNVs can be effectively used for successful delivery of anticancer agents into HCC cells and intrahepatic tumors. MNV-mediated targeted delivery of anticancer agents could be an efficient modality for the treatment of malignant HCC and might produce a great impact on anticancer therapy.
\end{abstract}

\section{Introduction}

Hepatocellular carcinoma (HCC) is a primary malignancy of the liver and is a global health problem. It is the third leading cause of cancer mortality worldwide, and advanced stages are associated with a dismal prognosis $[1,2]$. The pathogenesis of HCC involves several different potential etiologies and can be associated with diverse genetic alterations [3]. HCC is highly resistant to

Tushar Patel

patel.tushar@mayo.edu

1 Department of Transplantation, Mayo Clinic, Jacksonville, FL, USA

2 Department of Cancer Biology, Mayo Clinic, Jacksonville, FL, USA

3 Present address: Department of Hepatology, Kanazawa Medical University, Uchinada, Ishikawa 920-0293, Japan conventional chemotherapy or molecular-targeted therapies. The development of effective treatments for HCC is hampered by genetic and phenotypic changes that confer drug resistance or enhance cell survival. Treatment for HCC may be improved by the use of strategies that combine conventional anticancer agents and that concomitantly target other pathways to mitigate the effects of therapeutic resistance.

Alterations in non-coding RNA, such as microRNAs (miRNAs) and long noncoding RNAs offer new opportunities to target HCC [4]. Alterations in miRNA expression have been implicated in the pathogenesis and progression of HCC and other cancers, potentially acting through modulation of pathways involved in growth, survival, or therapeutic resistance $[5,6]$. The therapeutic potential of targeting cancer-associated miRNAs in $\mathrm{HCC}$ has been evaluated. Antisense oligonucleotides (ASO) targeting miR221 markedly reduced growth of orthotopic intrahepatic HCC, and increased survival rate in mice [7]. The coadministration of RNA-based therapies with anticancer 
agents offers an attractive strategy to improve treatment responses. Doxorubicin is a potent anticancer agent used for various cancers, including HCC, where it is used as part of chemoembolization or systemic protocols [8]. A downregulation of miR522 was observed in doxorubicin-resistant HT29 colon cancer cells, compared with parental cell lines [9]. Furthermore, over-expression of miR195 sensitized resistant cells to doxorubicin and enhanced cell apoptosis [10]. Thus, targeting selected RNA may enhance treatment responses to doxorubicin.

An ideal drug delivery method would selectively target tumor cells within the liver, while enabling coadministration of both RNA-targeting and anticancer therapies. By enabling targeted localization within tumors, and uptake by target cells, nanoparticle-based therapeutics could enhance efficacy, while simultaneously reducing side effects [11]. In this study, we sought to evaluate the use of biologically derived nanoparticles, or extracellular vesicles (EVs) for use in drug delivery. EVs are cell-derived vesicles that are enclosed within a lipid bilayer. Several types of EV have been described, such as exosomes, microvesicles, and oncosomes [12, 13]. Physiologically, EV can contribute to intercellular communication with shuttling of their content between cells [14]. Indeed, the transfer of RNA through EV can alter gene expression in recipient cells. These properties indicate that EVs have the ability to serve as therapeutic delivery vehicles to transfer RNA-based or anticancer agents to target cells $[15,16]$.

EVs are released from many different types of cells in the body, and can be found within biological fluids [17]. To evaluate the potential for the use of EV for therapeutic applications, we focused on the use of $\mathrm{EV}$ from milk as a scalable source of biological EVs. We developed approaches for isolation of membrane-enclosed nanovesicles from milk, evaluated their uptake by HCC cells in vitro, and assessed their application for use in delivery of doxorubicin and anti-miR221 oligonucleotides, and therapeutic efficacy of these against HCC cells in vitro and in vivo.

\section{Materials and methods}

\section{Isolation and characterization of nanovesicles from milk}

Vesicles were isolated from commercially obtained skim milk as follows. First the milk was mixed with phosphatebuffered saline (PBS) (10 ml milk: $14 \mathrm{ml}$ PBS) and centrifuged at $12,000 \times g$ for $30 \mathrm{~min}$ at $4{ }^{\circ} \mathrm{C}$. The supernatant was collected and filtered through a $0.45 \mu \mathrm{m}$ polyethersulfone filter to remove cell debris. The filtrate was then mixed with $1.5 \mathrm{ml}$ of $0.5 \mathrm{M}$ ethylenediaminetetraacetic acid (EDTA) (pH 8.0), followed by $3.0 \mathrm{M} \mathrm{HCl}$ and allowed to stand at $4{ }^{\circ} \mathrm{C}$ for $10 \mathrm{~min}$ to precipitate milk proteins followed by centrifugation at $500 \times g$ for $10 \mathrm{~min}$ at $4{ }^{\circ} \mathrm{C}$. The supernatant was collected. The $\mathrm{pH}$ of the supernatant was adjusted to 7.0 with $3.0 \mathrm{M} \mathrm{NaOH}$ and then centrifuged at $100,000 \times g$ for $70 \mathrm{~min}$ at $4{ }^{\circ} \mathrm{C}$. The pellet was resuspended in $1 \mathrm{ml}$ of $\mathrm{PBS}$ and centrifuged again at $100,000 \times g$ for $70 \mathrm{~min}$ at $4{ }^{\circ} \mathrm{C}$. The resulting pellet consisting of MNV was resuspended in $50 \mu \mathrm{l}$ of sterile PBS. The isolated MNVs were stained with DiI (\#V-22885, Thermo Fisher Scientific) and characterized using transmission electron microscopy and nanoparticle tracking analysis (NTA) (Nanosight, Malvern Instruments, Westborough, MA).

\section{Internalization of doxorubicin and miR221 ASO into milk nanovesicles}

Doxorubicin powder (\#D1515, Sigma, St. Louis, MO) was dissolved in water to a concentration of $2 \mathrm{mg} / \mathrm{ml} .1 \times 10^{11}$ particles of MNV in $900 \mu \mathrm{l}$ of PBS were mixed with $100 \mu \mathrm{l}$ of doxorubicin $(200 \mu \mathrm{g})$ and $5 \mu \mathrm{l}$ of lipofectamine 2000 (Invitrogen). The mixture was rotated overnight at $25 \mathrm{rpm}$ in an incubator at $37^{\circ} \mathrm{C}$. It was centrifuged at $100,000 \times g$ for $70 \mathrm{~min}$ at $4^{\circ} \mathrm{C}$ in an ultracentrifuge (Beckman Coulter, Optima L-80 XP) using SW 60 Ti rotor. The sediment was re-suspended in $2 \mathrm{ml}$ of sterile $\mathrm{PBS}$ and centrifuged again at $100,000 \times g$ for $70 \mathrm{~min}$ at $4{ }^{\circ} \mathrm{C}$. The residual pellet containing doxorubicin-loaded MNV (dox-MNV) was dissolved in $200 \mu \mathrm{l}$ of water. The concentration of doxorubicin in the supernatant and dox-MNV was determined using a spectrophotometer (Beckman DU-800) against a standard curve prepared with pure doxorubicin in water ranging from 10 to $50 \mu \mathrm{g} / \mathrm{ml}$ at $490 \mathrm{~nm}$. The dox-MNV was further characterized by NTA, measuring UV-Vis spectrum from 250 to $700 \mathrm{~nm}$, and concentration curve fitting ranging from 10 to $50 \mu \mathrm{g} / \mathrm{ml}$. ASO to miR221 were prepared and evaluated as described before [7]. About $1 \times 10^{11}$ particles of MNV in $900 \mu \mathrm{l}$ of PBS was mixed with $100 \mu \mathrm{l}$ of $5 \mathrm{nM}$ miR221 ASO and $5 \mu \mathrm{l}$ of lipofectamine 2000 and the mixture was rotated overnight at $25 \mathrm{rpm}$ in an incubator at $37^{\circ} \mathrm{C}$. The miR221 ASO-loaded MNV (anti-miR221-MNV) was isolated and purified as described above.

\section{Zeta potential (ZP) measurements}

ZP (electrostatic potential) of plain, doxorubicin-loaded or anti-miR221 -loaded MNV were measured using a Malvern Zetasizer (Malvern Instruments, Worcestershire, UK) and disposable zeta cells at a concentration of $1 \times 10^{10}$ particles of $\mathrm{MNV} / \mathrm{ml}$ employing zetasizer software. Prior to use, zeta cells were rinsed with water, followed by $70 \%$ ethanol, and dried well. A total of six samples were measured on each 
group. All the samples were programmed for four measurements and the mean value was obtained.

\section{Cellular uptake of MNV}

HepG2 cells (ATCC \#HB-8065) were procured from American Type Culture Collection (ATCC, Manassas, VA). The cells cultured in 96-well plates (10,000 cells/well) for $48 \mathrm{~h}$ and used for concentration dependent or kinetic uptake studies. Plain MNV and dox-MNV (final concentration $1.2 \times 10^{10}$ particles) in PBS were mixed with DiR solution (\#D-12731, Life Technologies, Grand Island, NY) to a final concentration of $4 \mu \mathrm{g} / \mathrm{ml}$. The tubes were covered with aluminum foil to protect from light and incubated in a shaking water bath at $37^{\circ} \mathrm{C}$ for $15 \mathrm{~min}$. It was centrifuged at $100,000 \times g$ for $70 \mathrm{~min}$ at $4{ }^{\circ} \mathrm{C}$ and the sediment containing MNV was collected and suspended in clear media. For these studies, vesicle-depleted media was used as described previously [18]. For concentration-dependent uptake studies, the culture media was replaced with media containing DiR-labeled MNV (plain or doxorubicin-loaded) at final concentrations of $1.2,0.6,0.3$, and $0.15 \times 10^{10}$ particles $/ \mathrm{ml}$ (the respective final concentrations of doxorubicin in $\mathrm{MNV}$ were $6,3,1.5$, and $0.75 \mu \mathrm{g} / \mathrm{ml}$ ), and transferred to a $\mathrm{CO}_{2}$ incubator. Control sets were also prepared with unlabeled plain MNV, unlabeled dox-MNV, and cells treated with DiR dye alone in PBS. Kinetic studies of uptake were performed using plain or dox-MNV at a concentration of $1.2 \times 10^{10}$ particles $/ \mathrm{ml}(6 \mu \mathrm{g} / \mathrm{ml}$ doxorubicin, final concentration in MNV and media), with samples obtained at various time points upto $24 \mathrm{~h}$. At the indicated time points, the media was aspirated; cells were washed twice with PBS, and scanned on LI-COR Odyssey infrared fluorescent imaging system (LI-COR, Lincoln, NE). The IR images were quantified using Image-Pro Plus software (Media cybernetics, Rockville, MD). Data are presented as square microns.

\section{Internalization of dox-MNV and anti-miR221-MNV into HepG2 cells}

MNV containing doxorubicin (dox-MNV), antisense oligonucleotide to miR221 (anti-miR221-MNV) singly or in combination (dox-anti-miR221-MNV) were labeled with PKH67 (\#MINI67, Sigma-Aldrich, St. Louis, MO). HepG2 cells were cultured on collagen-coated glass slides (Nunc Lab-Tek, Thermo Scientific) in Dulbecco's modified Eagle's medium (DMEM) supplemented with $10 \%$ fetal bovine serum (FBS) and antibiotics in a humidified incubator containing $5 \% \mathrm{CO}_{2}$ on air at $37^{\circ} \mathrm{C}$. After $48 \mathrm{~h}$, the media was replaced with regular media suspended with dox-MNV (doxorubicin $400 \mathrm{ng} / \mathrm{ml}$ media), anti-miR221MNV (500 pmoles, final conc.) or dox-anti-miR221-MNV along with appropriate controls. The concentration of MNV was $3.2 \times 10^{10}$ particles $/ \mathrm{ml}$ media in all cases. After incubation for $48 \mathrm{~h}$, the media was removed, cells were washed twice with PBS, fixed with $4 \%$ paraformaldehyde, and treated with tetramethylrhodamine-conjugated phalloidin for $30 \mathrm{~min}$ in dark. The cells were further washed and mounted with Prolong Gold antifade reagent containing nuclear stain DAPI (\#P36941, Life Technologies, Grand Island, NY). The slides were then examined under a fluorescence microscope (Olympus BX51, Tokyo, Japan) connected with a DP71 Olympus digital camera to study the internalization and cellular effect of dox-MNV, antimiR221-MNV, and dox-anti-miR221-MNV and photographed.

\section{Kinetic studies of cytotoxicity of Dox-MNV and anti- miR221-MNV on HepG2, Hep3B, and PLC/PRF/5 cells}

In order to study the effect and rate of cytotoxicity and cell death during treatment with doxorubicin and dox-MNV, as well as anti-miR221 and anti-miR221-MNV on various human HCC cell lines, we cultured HepG2, Hep3B, and $\mathrm{PLC} / \mathrm{PRF} / 5$ cells in 96-well plates at a concentration of 10,000 cells/well. Hep3B (ATCC, \#HB-8064) and PLC/ PRF/5 (ATCC \#CRL-8024) cells were procured from American Type Culture Collection (ATCC, Manassas, VA). After $24 \mathrm{~h}$ incubation, the media was replaced with clear media containing regular doxorubicin (\#D1515, Sigma, St. Louis, MO) or Dox-MNV at a final concentration of $1 \mu \mathrm{M}$ $(580 \mathrm{ng} / \mathrm{ml}$ media) or anti-miR221 or anti-miR221-MNV (500 pmoles, final concentration in the media). Untreated cells and cells treated with plain MNV served as controls. At 24,48 , and $72 \mathrm{~h}$ after the treatment, $20 \mu \mathrm{l}$ of MTS reagent (\#G3580, Promega, Madison, WI) was added to the wells. The plates were incubated for $3 \mathrm{~h}$ at $37{ }^{\circ} \mathrm{C}$ in a $\mathrm{CO}_{2}$ incubator and the color intensity measured on a microplate reader (FLUOstar Omega, BMG Labtech, Cary, NC) at 490 $\mathrm{nm}$.

\section{Cytotoxic effect of low doses of doxorubicin and Dox-MNV on HCC cells}

In order to study whether there is a difference in the effect of usual doxorubicin and Dox-MNV at lower doses, we tested the cytotoxic effect of two reduced doses of usual doxorubicin and Dox-MNV (500 and $750 \mathrm{nM}$ ) on HepG2, Hep3B, and PLC/PRF/5 HCC cells. The cells were cultured in 96-well plates at a concentration of 10,000 cells/well and treated with doxorubicin or Dox-MNV at concentrations of 500 and $750 \mathrm{nM}$ (final concentration in the media), and measured the rate of inhibition of cell growth at $72 \mathrm{~h}$ after the treatment using MTS reagent (\#G3580, Promega, Madison, WI) as described above. 


\section{Confocal microscopic studies for tracking and interaction of MNV with subcellular organelles}

PLC/PRF/5 HCC cells were cultured on collagen-coated glass slides for $24 \mathrm{~h}$. About $1 \times 10^{11}$ particles of MNV in $1 \mathrm{ml}$ of PBS were labeled with PKH67, purified using ultracentrifugation, suspended in clear media, and treated with the cells for $24 \mathrm{~h}$. The media was removed and cells were washed twice with Hank's balanced salt solution (HBSS) containing calcium and magnesium, followed by incubation for $30 \mathrm{~min}$ in HBSS containing $200 \mathrm{nM}$ oxidized rosamine-based mitotracker (\#M7512, Molecular Probes, Eugene, OR). Cells were then washed twice with PBS, and fixed using $4 \%$ paraformaldehyde for $10 \mathrm{~min}$. For staining Golgi apparatus, the MNVs were labeled with DiI (\#V-22885, Thermo Fisher Scientific). The cells were washed with PBS and incubated overnight with rabbit TGN46 (Trans-Golgi network protein, $46 \mathrm{kDa}$ ) antibody (\#T7576, Sigma, St. Louis, MO) followed by second antibody conjugated with Alexa Flour 488. The cells were washed and the nuclei were stained with $2 \mu \mathrm{g} / \mathrm{ml}$ solution of Hoechst (\#H3570, Invitrogen) for $10 \mathrm{~min}$ and finally mounted with Prolong Gold antifade reagent (\#P36934, Molecular Probes, Life Technologies) and allowed to dry for $30 \mathrm{~min}$ in dark. The slides were then examined on a Carl Zeiss LSM 510 Meta confocal microscope using Z-stack imaging.

For cellular localization studies of ASO delivered by MNV, miR221-ASO were biotinylated using Pierce RNA 3' end biotinylation kit (\#20160 Thermo Scientific), purified, and evaluated using dot-blot hybridization. $1 \times 10^{11}$ particles of MNV in $1 \mathrm{ml}$ of PBS were loaded with 20 pmol of biotinylated miR221-ASO using $2 \mu \mathrm{l}$ of lipofectamine 2000 and purified as described above. After $24 \mathrm{~h}$, the cells were fixed with $4 \%$ paraformaldehyde for $10 \mathrm{~min}$, washed, and incubated with rabbit early endosomal antigen 1 (EEA1) antibody (\#2411, Cell Signaling Technology, Danvers, MA) overnight at $4{ }^{\circ} \mathrm{C}$. The cells were washed and simultaneously treated with goat anti-rabbit second antibody conjugated with Alexa Flour 488, streptavidin-conjugated Alexa Flour 647, and appropriately diluted Hoechst 33342 (\#H3570, Invitrogen) for $1-2 \mathrm{~h}$ at room temperature in dark. The slides were washed five times in PBS under dim light, mounted with aqua-Poly/Mount (\#18606, Polysciences, Warrington, PA) and allowed to dry for $30 \mathrm{~min}$ in dark. The slides were then examined on a confocal microscope (Carl Zeiss LSM 510) using Z-stack imaging with simultaneous use of DAPI/FITC/ Alexa 647 lasers for Hoechst, EEA1, and biotinylated antimiR221-MNV, respectively, and the images were saved.

\section{Induction of intrahepatic tumors in athymic nude mice}

The animal experiments were carried out in accordance with the National Institutes of Health Guide for the Care and Use of Laboratory Animals (NIH Publications No. 8023, revised 1978) and in accordance with an Institutional Animal Care and Use Committee (IACUC) approved protocol. Eightweek old, athymic nude mice were procured from Jackson Laboratories (Farmington, CT). PLC/PRF/5 HCC cells (ATCC \#CRL-8024) were stably transfected with a mammalian expression vector carrying the luciferase gene phCMV-FSR (\#P013400 Genlantis, San Diego, CA) and were propagated in DMEM containing G-418 $(500 \mu \mathrm{g} / \mathrm{ml})$. The cells were highly homogeneous and propagated from a single cell colony. Cell cultures in log phase were harvested, washed twice in serum-free medium, counted, and suspended in serum-free DMEM containing 50\% highly concentrated Matrigel without phenol red (BD Biosciences, San Jose, CA). Around $1 \times 10^{6}$ cells in $20 \mu \mathrm{l}$ suspension were injected through the diaphragmatic surface into the large left lobe after surgical exposure of the liver. Bioluminescence was monitored every week in the tumor implanted animals using IVIS in vivo imaging system (Xenogen IVIS-200, Perkin Elmer, Hopkinton, MA) after injecting $100 \mu \mathrm{l}$ of D-luciferin (Gold Biotech, St. Louis, $\mathrm{MO})$ in sterile saline $(50 \mathrm{mg} / \mathrm{ml})$ intraperitoneally to activate the luciferin-luciferase bioluminescence system. When the signals exceeded $1 \times 10^{7}$ photons/s, the animals were injected through tail vein either with dox-MNV $(400 \mu \mathrm{g}$ dox/kg body weight) or anti-miR221-MNV (500 nmoles/kg body weight) or both in combination (dox-anti-miR221MNV) or plain MNV (controls) thrice a week for 4 weeks. The animals were maintained for another 1 week and the bioluminescence was measured before sacrifice.

\section{Statistical analysis}

Arithmetic mean and standard deviation (mean $\pm \mathrm{SD}$ ) were calculated for all the data. The data were analyzed by either using one-way analysis of variance (ANOVA) or multiple group comparison depending on the situation. Paired Student's $t$-test was also used whereever applicable. Control mean values were compared with experimental mean values on different time points using least significant difference method. A value of $P<0.05$ was considered as statistically significant.

\section{Results}

\section{Characteristics of nanovesicles isolated from skim milk}

The nanovesicles obtained from skim milk were examined using fluorescence microscopy after staining with DiI and using TRITC filter (Fig. 1a) (Olympus BX51, Tokyo, Japan), or using transmission electron microscopy (Fig. 1b). 


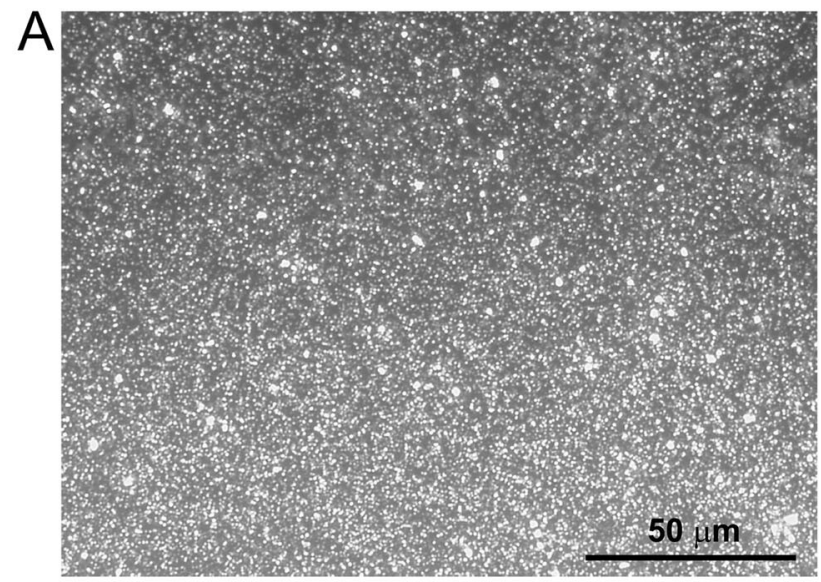

Dil staining of MNV (TRITC $\times 400)$

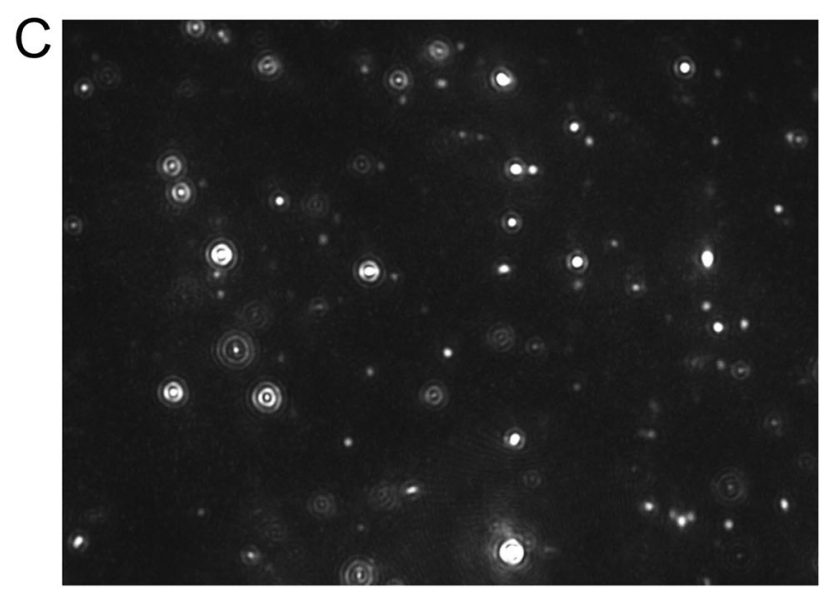

Still image of the laser scattering of MNV

Fig. 1 Isolation and characterization of milk nanovesicles (MNV). a DiI staining of MNV (TRITC $\times 400)$. b Transmission electron microscopic image of MNV. c Image of laser scattering of MNV during

Most of the MNVs were in the size range of $100-200 \mathrm{~nm}$. Further validation of size and quantitation was performed using NTA (Fig. 1c,d). A laser beam was passed through the MNV in a chamber and the particles in suspension in the path of the beam were visualized using a microscope mounted with a camera connected to a computer. By this approach, more than $90 \%$ of $\mathrm{MNV}$ were noted to be between the size of 100 and $200 \mathrm{~nm}$. Thus, the MNV isolation protocol provides a population of EV within the size range of exosomes, or very small microvesicles.

\section{Characterization of doxorubicin-loaded MNV}

MNV were loaded with doxorubicin, purified using ultracentrifugation, and characterized (Fig. 2a). We observed that the supernatant was very clear without any trace of doxorubicin after a second wash. After NTA, we noted that doxorubicin-loaded MNV had a peak particle size of $175 \mathrm{~nm}$ (Fig. 2b). Additional peaks were observed at 265, 345, and

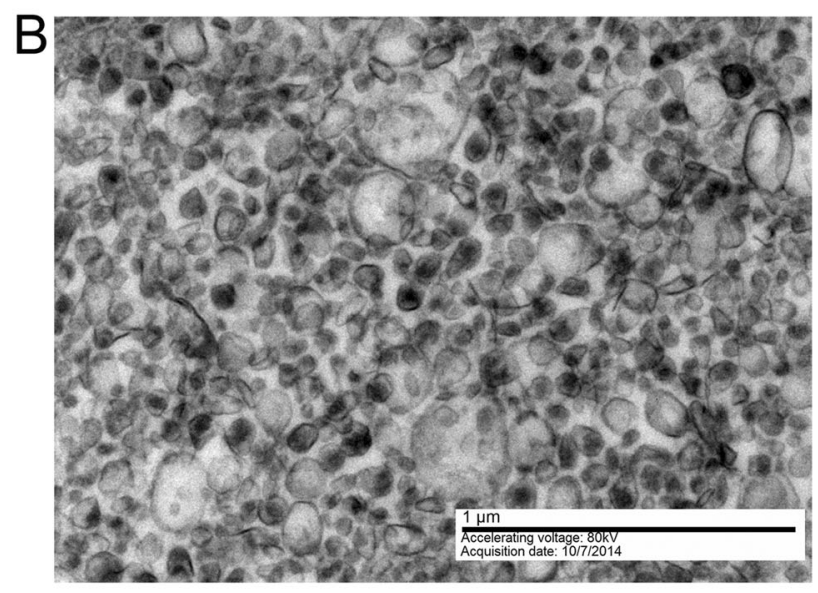

Transmission Electron Microscopic image of MNV

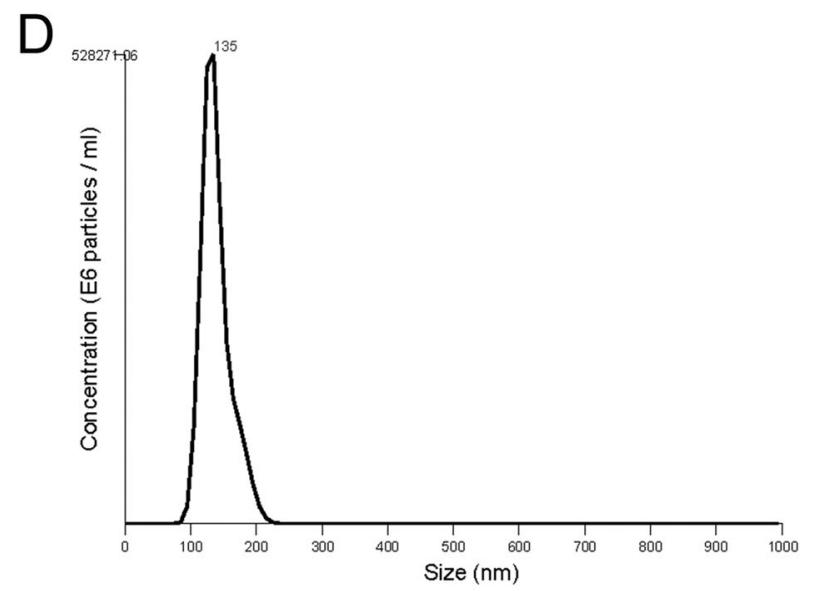

Nanoparticle Tracking Analysis (NTA) of MNV

nanoparticle tracking analysis (NTA). d Size/concentration analysis of purified MNV using NanoSight depicting high-resolution finite track length adjustment (FTLA) algorithm graph

$475 \mathrm{~nm}$. These would be consistent with formation of clusters or aggregation of dox-MNV induced either during the loading process, or by the presence of doxorubicin within the MNV. UV-visible spectrophotometric scanning of pure doxorubicin and dox-MNV revealed that the peak at $490 \mathrm{~nm}$ for dox-MNV was identical to that of pure doxorubicin (Fig. 2c). In all samples, the concentration of doxorubicin was $50 \mu \mathrm{g} / \mathrm{ml}$. Even though the peaks were identical, we noted an upward spectral shift associated with the presence of MNV. Curve fitting of optical density against concentration of dox-MNV, ranging from a doxorubicin concentration of $10-50 \mu \mathrm{g} / \mathrm{ml}$ produced a straight line graph indicating dox-MNV follows Beer-Lambert law (Fig. 2d).

\section{Alteration of ZP after internalization of anticancer agents in MNV}

The ZP describes the strength of the particle electrical barrier measured in terms of an electrical potential. $\mathrm{ZP}$ 

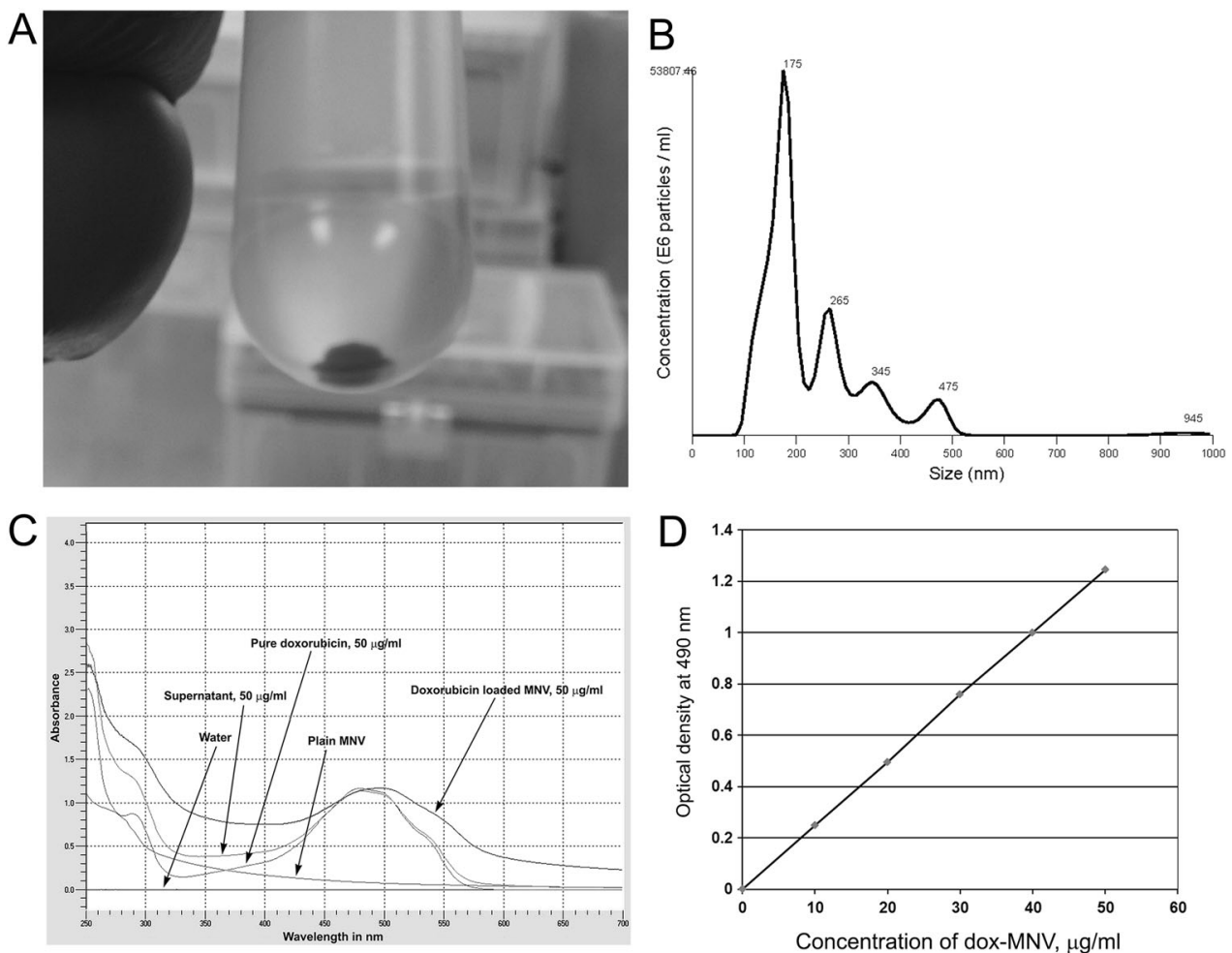

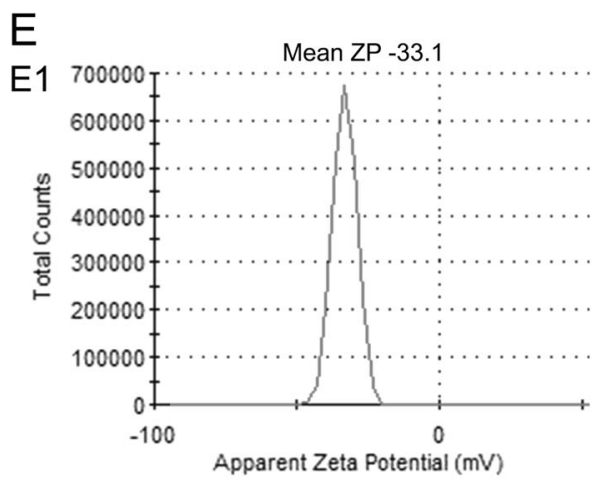

Plain MNV

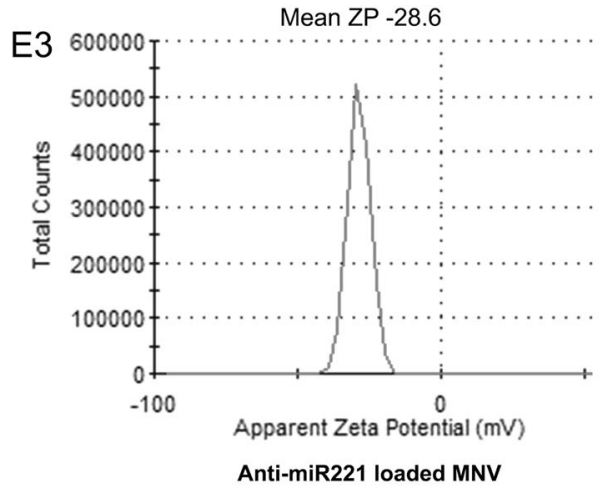

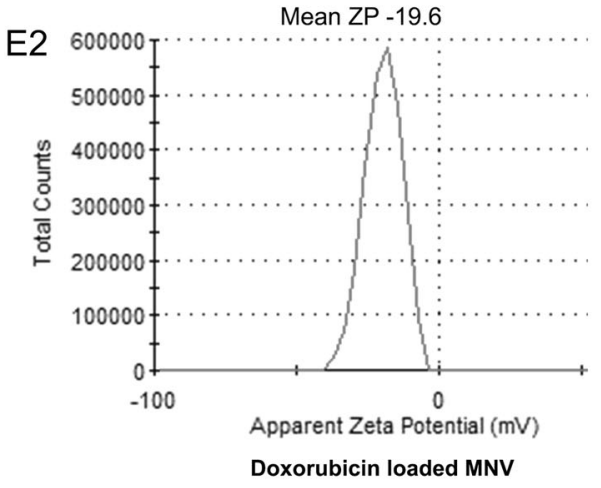

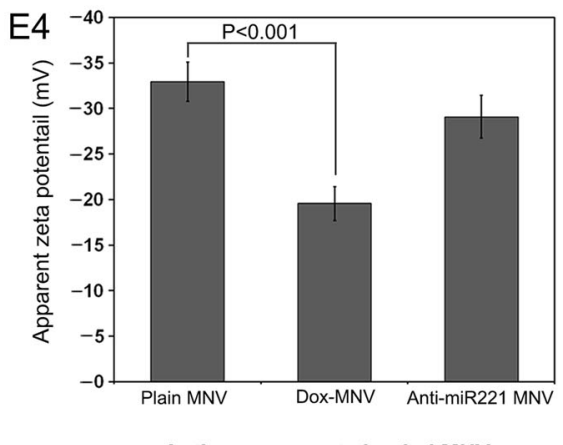

Anticancer agents loaded MNVs 
Fig. 2 Characterization of doxorubicin-loaded MNV (dox-MNV). a Dox-MNV after ultracentrifugation. The supernatant was very clear without a trace of doxorubicin after second wash. b Size/concentration analysis of dox-MNV using NanoSight. c UV-visible spectrum of pure doxorubicin and dox-MNV at a fixed concentration of $50 \mu \mathrm{g} / \mathrm{ml}$. d Concentration curve fitting of dox-MNV ranging from 10 to $50 \mu \mathrm{g} /$ $\mathrm{ml}$ at $490 \mathrm{~nm}$. eZeta potential measurements of plain MNV and after internalization of doxorubicin and anti-miR221. e1 Plain MNV and e2 dox-MNV. e3 Anti-miR221 loaded MNV. e4 Quantitative analysis of zeta potential measurements. Data are mean \pm SD of five samples in each group

measurements of plain MNV and after internalization of doxorubicin and anti-miR221 are presented in Fig. 2e. Plain MNV have an apparent ZP of $-33.1 \mathrm{mV}$ (Fig. 2e1), but MNV loaded with doxorubicin have a significant decrease $(P<0.001)$ of $\mathrm{ZP}$ with an apparent $\mathrm{ZP}$ of $-19.6 \mathrm{mV}$ (Fig. 2e2). In contrast, even though there was a decrease of $\mathrm{ZP}(-28.6 \mathrm{mV})$ in $\mathrm{MNV}$ after internalization of antimiR221, this was not significantly different from the ZP of plain MNV (Fig. 2e3). Quantitative analysis of ZP measurements identified a significant reduction of $\mathrm{ZP}$ after loading of MNV with doxorubicin while no change was observed after internalization of anti-miR221 (Fig. 2e4). A reduction in ZP may reflect an increased tendency of particle aggregation. Thus, these results suggest that antimiR221-loaded MNV may have less tendency to aggregation compared with dox-MNV.

\section{Cellular uptake of plain and dox-MNV by HepG2 cells}

We next examined the ability of target HCC cells to take up MNV. Studies in HepG2 cells incubated with either plain, or doxorubicin-loaded MNV revealed no difference in the rate of cellular uptake of either of these types of MNV (Fig. 3a1). The duplicate samples with the same concentration of either loaded or plain MNV produced exactly similar images indicating complete uptake of all loaded MNV by HepG 2 cells. The lack of a background signal with DiR dye alone indicated that the entire observed signal is from the DiR-labeled MNVs. Since there was no signal, the images of unlabeled plain MNV and unlabeled dox-MNV are not shown. The quantitative evaluation of the rate of uptake of plain and dox-MNV by HepG2 cells revealed a sequential increase in the rate of uptake in parallel with the increase of both plain MNV and dox-MNV (Fig. 3a2). The quantitative values were significantly different $(P<0.001)$ between the groups in both plain and dox-MNV.

\section{Kinetics of the uptake of plain and dox-MNV by HepG2 cells}

Kinetic uptake studies of plain and dox-MNV were performed in HepG2 cells. There was sequential increase in the rate of uptake, with about $50 \%$ of both plain and dox-MNV taken up by HepG2 cells within $1 \mathrm{~h}$, and maximum uptake by $4 \mathrm{~h}$ (Fig. 3b1). The rate of uptake of MNV was not influenced by loading with doxorubicin (Fig. 3b2).

\section{Effect of dox-MNV and anti-miR221 MNV on HepG2 cells}

The effects of anticancer agents, doxorubicin, and antimiR221 delivered through MNV into HepG2 cells are presented in Fig. 4. Figure 4a depicts untreated HepG2 cells stained with TRITC-phalloidin and DAPI. Treatment with unlabeled MNV did not produce any alteration in HepG2 cells (Fig. 4b). Internalization of PKH-labeled MNV demonstrated perinuclear distribution in the cytoplasm (Fig. 4c). Z-stack confocal microscopy revealed that the labeled MNV was well dispersed in the cytoplasm covering most areas and did not produce any alteration either in the nucleus or cytoplasm of the cells (image not shown). When studies performed using PKH67-labeled dox-MNV, internalization of MNV was accompanied by alterations in the cytoplasm along with nuclear blebbing indicative of apoptosis (Fig. 4d). Likewise, uptake of MNV loaded with miR221 ASO was associated with nuclear disintegration consistent with cell death (Fig. 4e). Internalization of large numbers of PKH67-labeled anti-miR221-MNV into the cytoplasm of HepG2 cells is conspicuous in the image (Fig. 4e). When HepG2 cells were incubated with MNV loaded with both doxorubicin and anti-miR221 ASO, nuclear disintegration and apoptosis was observed in almost all cells (Fig. 4f). These results indicate that delivery of anticancer agents using MNV is effective, and feasible, and moreover that this approach can be used for combination treatment modalities that can increase effectiveness over single agents to induce tumor cell death.

\section{Effect of doxorubicin, dox-MNV, anti-miR221, and anti-miR221-MNV on HepG2, Hep3B, and PLC/PRF/5 HCC cells}

In order to evaluate whether there is a difference in the rate of cell death in response to doxorubicin when administered via MNV, we determined cell viability over time after treatment with regular doxorubicin and dox-MNV in three different HCC cell lines, HepG2, Hep3B, and PLC/PRF/5 cells. Similar treatment has been carried out with antimiR221 and anti-miR221-MNV also. There was no difference in the rate of cell death either between regular doxorubicin and dox-MNV or between anti-miR221 and antimiR221-MNV in all the three cell lines studied (Fig. 5). Hep3B cells were more susceptible to doxorubicin compared to the other two cell lines studied. In Hep3B cells, there was a significant increase $(P<0.0001)$ in the cell 

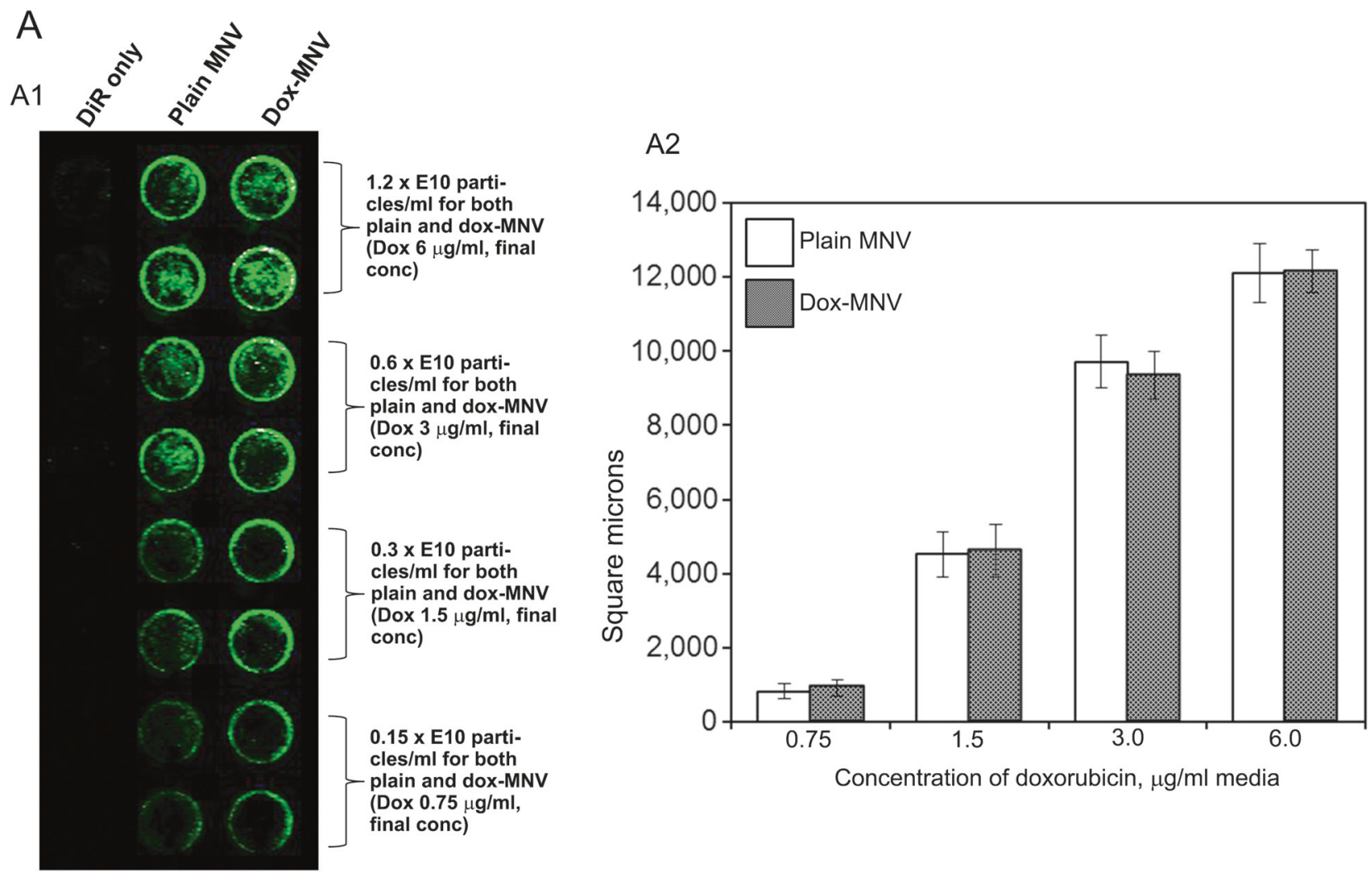
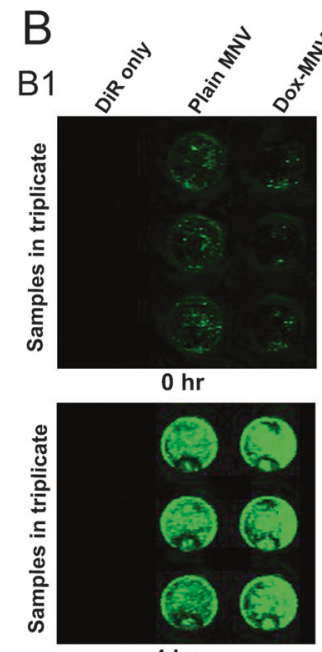

$4 \mathrm{hr}$

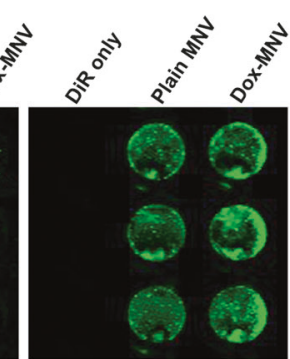

$1 \mathrm{hr}$

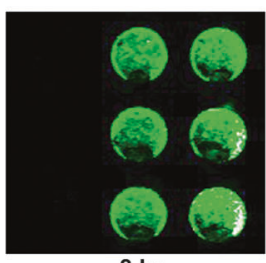

$8 \mathrm{hr}$
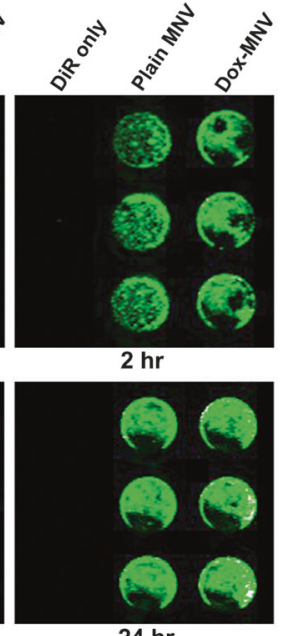

$24 \mathrm{hr}$

Fig. 3 a Rate of uptake of plain and dox-MNV by HepG2 cells. a1 HepG2 cells were cultured in 96-well plates at a concentration of 10,000 cells/well. After $48 \mathrm{~h}$, the media was replaced with clear media containing DiR-labeled plain and dox-MNV at various concentrations. After $24 \mathrm{~h}$, the media was aspirated; cells were washed twice with PBS, and scanned on LI-COR Odyssey infrared fluorescent imaging system. A2 Quantitative evaluation of the rate of uptake of plain and dox-MNV by HepG2 cells. The IR images were quantified using Image-Pro Plus software and the data are presented as square microns $(n=8)$. bKinetic studies of the uptake of plain and dox-MNV by HepG2 cells. b1 DiR-labeled plain and dox-MNV at a concentration of

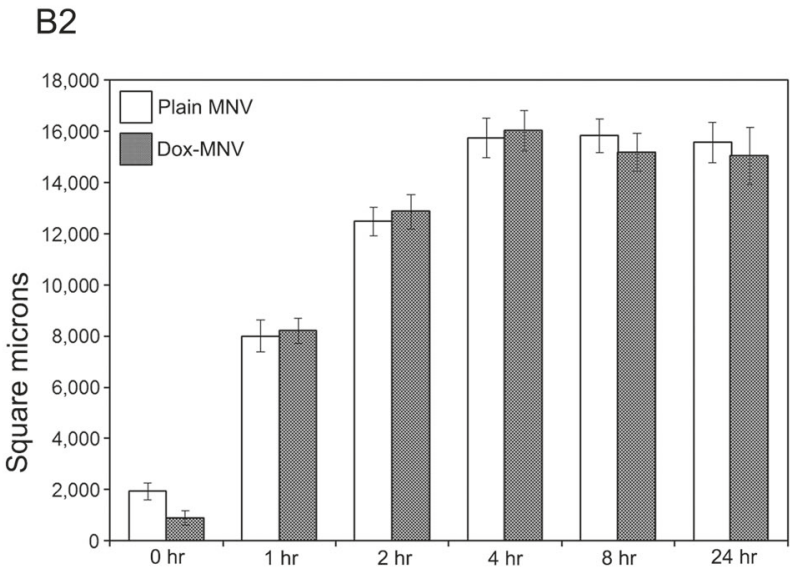

$1.2 \times \mathrm{E} 10$ particles $/ \mathrm{ml}(6 \mu \mathrm{g} / \mathrm{ml}$ doxorubicin, final concentration in MNV and media) were mixed with clear media and added to $48 \mathrm{~h}$-old HepG2 cultures in 96-well plates. At $0,1,2,4,8$, and $24 \mathrm{~h}$, the media was aspirated, cells were washed twice with PBS, and scanned on LICOR Odyssey infrared fluorescent imaging system. There was no difference in the rate of uptake between plain and dox-MNV at any time point studied. b2 Quantitative evaluation of the kinetics of the uptake of plain and dox-MNV by HepG2 cells. The IR images were quantified using Image-Pro Plus software and the data are presented as square microns $(n=8)$ 

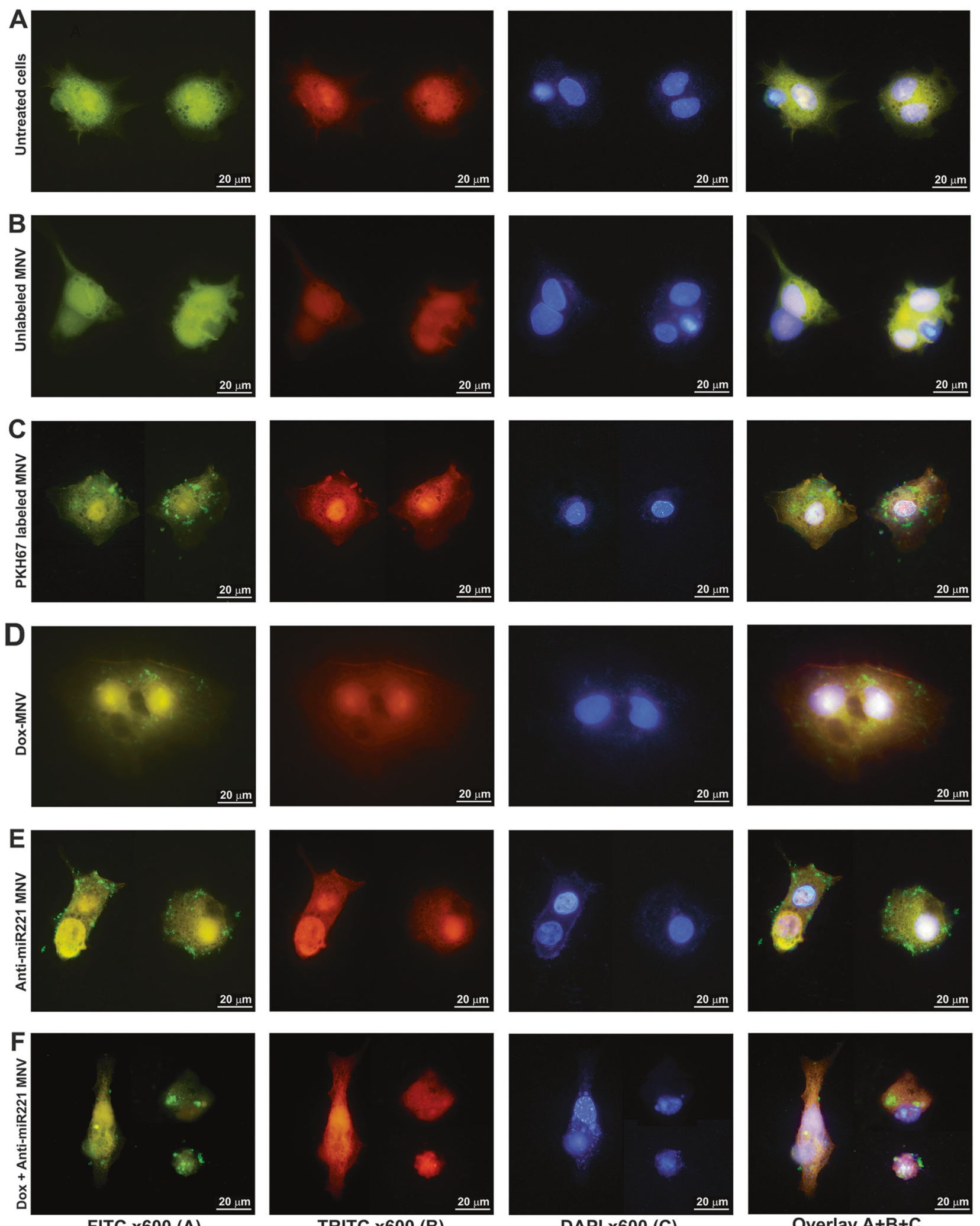

death at $72 \mathrm{~h}$ compared to $24 \mathrm{~h}$ (Fig. 5a2). In contrast, a significant increase $(P<0.0001)$ was observed in cell viability at $72 \mathrm{~h}$ compared to either 24 and $48 \mathrm{~h}$ in PLC/PRF/5 cells (Fig. 5a3). These results indicate that the concentrations studied are inadequate to kill aggressive and rapidly dividing cells, such as PLC/PRF/5. The rate of cell death in 
Fig. 4 Effect of dox-MNV and anti-miR221-MNV on HepG2 cells. The cells were cultured on collagen-coated glass slides for $48 \mathrm{~h}$ and then treated with media containing dox-MNV $(400 \mathrm{ng} / \mathrm{ml})$ or antimiR221-MNV (500 pmoles, final concentration) or dox-anti-miR221MNV and incubated for another $48 \mathrm{~h}$. a Control cells $(\times 600)$. b Cells treated with plain MNV $(\times 600)$. c Cells treated with PKH67-labeled MNV $(\times 600)$. Perinuclear distribution of MNV in the cytoplasm. d Cells internalized with PKH67-labeled dox-MNV $(\times 600)$. Alterations in the cytoplasm and nuclear blebbing indicating apoptosis. e Cells treated with PKH67-labeled anti-miR22-MNV $(\times 600)$. Nuclear disintegration consistent with cell death. f Cells internalized with both doxMNV and anti-miR221-MNV (×600). Nuclear disintegration and apoptosis in almost all cells.

HepG2 cells was not significantly different at 48 or $72 \mathrm{~h}$ compared to $24 \mathrm{~h}$ (Fig. 5A1). There was no difference in the rate of cell growth between anti-miR221 and antimiR221-MNV at 24,48 , or $72 \mathrm{~h}$ in all the three cell lines studied (Fig. 5b1-b3). In the case of PLC/PRF/5 cells, there was a significant increase in the rate of cell growth at $72 \mathrm{~h}$ compared to 24 and $48 \mathrm{~h}$ (Fig. 5b3), which could be attributed to the virulency of the cell line. However, such increase was absent in the case of HepG2 and Hep3B cells.

\section{Effect of low doses of doxorubicin and dox-MNV on HCC cells}

Since we did not notice any difference between the effect of regular doxorubicin and dox-NMV at $1 \mu \mathrm{M}$ level, we evaluated the cytotoxic effect of usual doxorubicin and doxNMV at two lower doses (500 and $750 \mathrm{nM}$ ) on HepG2, Hep3B, and PLC/PRF/5 HCC cells. The results are depicted in Fig. 6. At $500 \mathrm{nM}$, a significant difference $(P<0.001)$ was noticed in the effect of dox-MNV compared to regular doxorubicin in the case of HepG2 and Hep3B cells (Fig. 6a). However, the difference was not significant in the case of PLC/PRF/5 cells, which could be attributed to the virulency of PLC/PRF/5 cells. The rate of inhibition of cell growth was significantly decreased at $500 \mathrm{nM}$ compared to $750 \mathrm{nM}$ for both regular doxorubicin and doxMNV.

At $750 \mathrm{nM}$, the difference in cytotoxic effect between regular doxorubicin and dox-MNV was not significant in any cell line studied (Fig. 6b). However, the rate of inhibition of cell growth was significantly high $(P<0.001)$ compared to $500 \mathrm{nM}$ in all the cell lines studied. The inhibition of cell growth followed the same pattern as observed when the cells were treated with regular doxorubicin and dox-MNV at $1 \mu \mathrm{M}$. This experiment provided evidence that when HCC cells are treated with lower doses of dox-MNV, the rate of inhibition of cell growth is higher compared to the treatment with regular doxorubicin. This could be explained by the fact that all the dox-MNV is directly going inside the cells within $4 \mathrm{~h}$ of treatment. However, in the case of regular doxorubicin, a portion could be dispersed in the culture medium and may not be fully effective for the inhibition of cell growth.

\section{Interaction of MNV with subcellular organelles}

In order to track the cellular fate of internalized MNV and also to study the interaction and co-localization of MNV with subcellular organelles, we used confocal microscopy of MNV labeled with fluorescent conjugates and simultaneous labeling of subcellular organelles using specific fluorescent markers. We did not detect any interaction between rosamine-labeled mitochondria (Fig. 7a) or TGN46-labeled Golgi apparatus (Fig. 7b) and PKH67 or DiI labeled MNV following their uptake in PLC/PRF/5 cells. The absence of interaction between internalized MNV and these organelles was further verified by confocal Zstacking (images not shown). Results of MNV localization with early endosome are depicted in Fig. 7. Early endosomes were identified in a perinuclear distribution (Fig. 7c). We next examined the uptake of MNV loaded with therapeutic agents and their interactions with early endosomes in PLC/PRF/5 cells. Biotin-labeled anti-miR221 oligonucleotides-loaded MNV were internalized into PLC/ PRF/5 HCC cells and detected using streptavidinconjugated Alexa Flour 647 on a confocal microscope and are visible as deep red (Fig. 7d). Confocal microscopy did not support direct co-localization of loaded MNV with early endosomes.

\section{Treatment of intrahepatic tumors with MNV-loaded anti-cancer agents}

In order to evaluate the efficacy of dox-MNV, anti-miR221$\mathrm{MNV}$, and both in combination (dox-anti-miR221-MNV) on tumor regression, we induced intrahepatic tumors in athymic nude mice and treated with the anti-cancer agents systemically (Fig. 8). Tumor cells stably transfected with a mammalian expression vector carrying the luciferase gene were implanted into the liver and monitored using IVIS in vivo imaging system after injecting with D-luciferin. Luminescence intensity was exceeded $1 \times 10^{7}$ photons/s within 15 days. Untreated mice developed large intrahepatic tumors, which also metastasized to other organs. There was significant decrease in the size of the tumor and the amount of photons in the animals treated with both dox-MNV and anti-miR221-MNV through tail vein injections (Fig. 8a). Marked reduction was observed in tumor size in the animals treated with the anti-cancer agents in combination. The amount of photons decreased below the level at which the treatment was started $\left(1 \times 10^{7}\right.$ photons $\left./ \mathrm{s}\right)$. Besides, the effect of the treatment of both the agents in combination was synergistic. Metastasis was completely absent in all the 

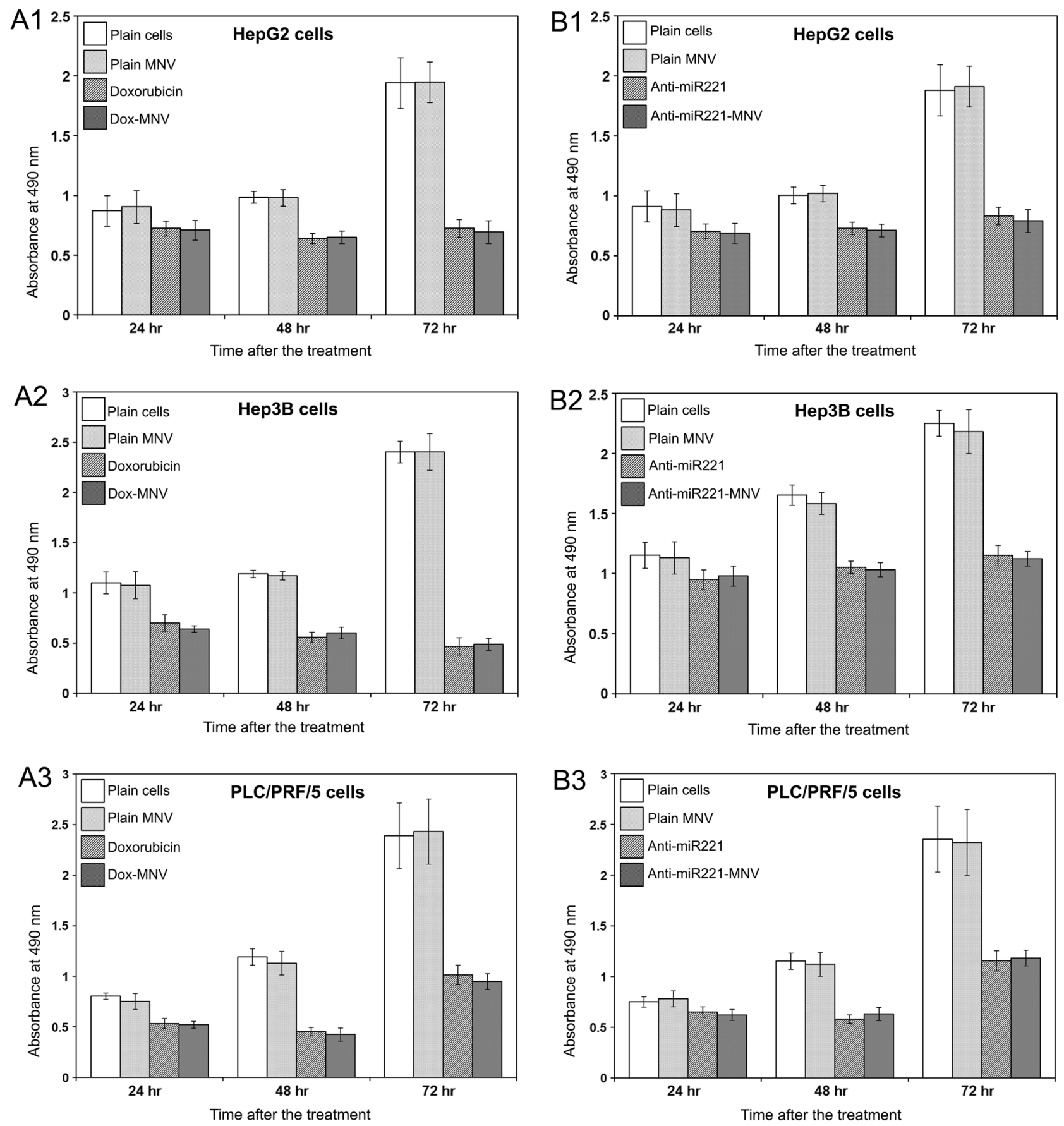

Fig. 5 Effect of anticancer agents on cytotoxicity and cell death. HepG2, Hep3B, and PLC/PRF/5 HCC cells on culture were treated with regular doxorubicin and dox-MNV (final concentration $1 \mu \mathrm{M}$ $(580 \mathrm{ng}) / \mathrm{ml}$ media) or anti-miR221 and anti-miR221-MNV (500 pmoles, final concentration in the media) for 24,48 , and $72 \mathrm{~h}$ and assayed for cell viability. There was no difference in the rate of cell

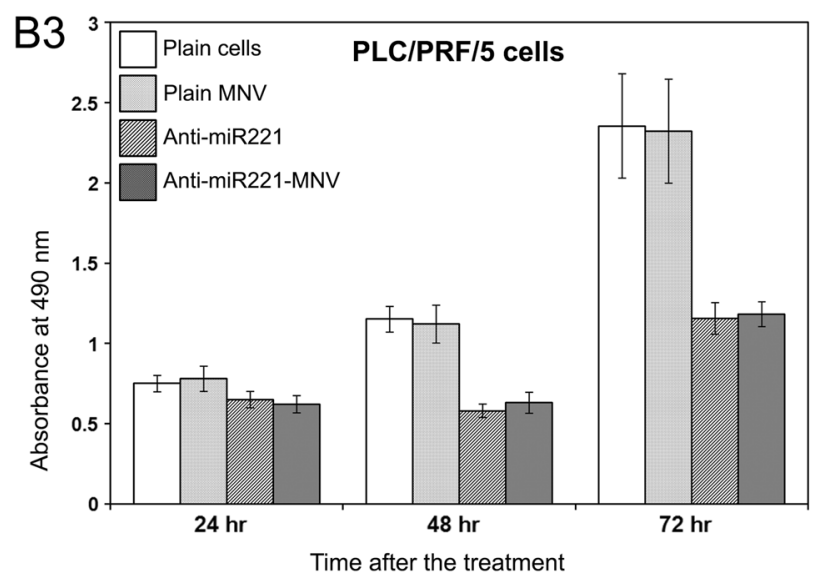

death between regular doxorubicin and dox-MNV (a1-a3) or antimiR221 and anti-miR221-MNV (b1-b3) in all the three cell lines studied. The rate of cell death was significantly higher with doxorubicin compared to anti-miR221 treatment. The data are mean $\pm \mathrm{SD}(n$ $=8)$

animals treated with either dox-MNV or anti-miR221-MNV or in combination. Figure $8 \mathrm{~b}$ represents the mean and deviation of bioluminescence photons measured using IVIS in five animals in each group. There was significant decrease $(P<0.0001)$ in the amount of photons emitted

from intrahepatic tumors in the animals treated with either dox-MNV or anti-miR221-MNV compared to controls. The animals treated with both the agents in combination depicted marked decrease in the amount of photons compared to the treatment singly (Fig. 8b). 

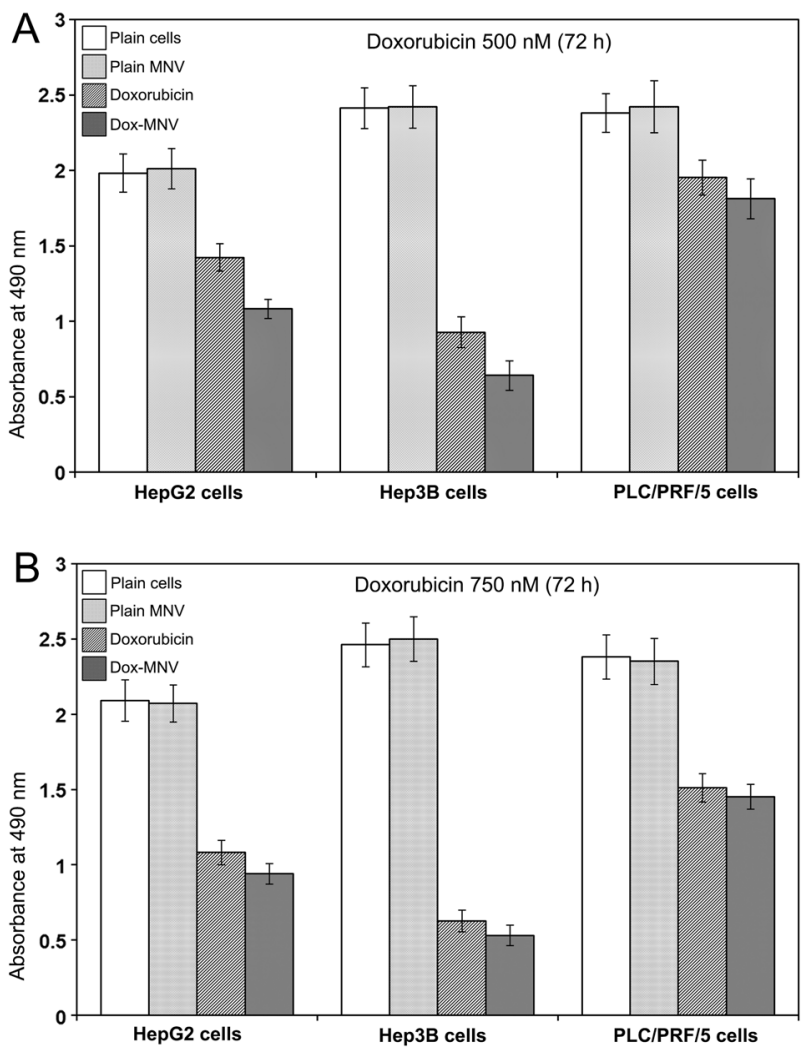

Fig. 6 Effect of doxorubicin and dox-MNV on HCC cells. HepG2, Hep3B, and PLC/PRF/5 HCC cells were treated with regular doxorubicin and dox-MNV at final concentrations of 500 and $750 \mathrm{nM}$ for 72 $\mathrm{h}$ and assayed for cell viability. There was significant difference in the rate of cell death between regular doxorubicin and dox-MNV at 500 $\mathrm{nM}$ in the case of HepG2 and Hep3B cells (a). The difference was not significant at $750 \mathrm{nM}(\mathbf{b})$. The data are mean $\pm \mathrm{SD}(n=8)$

\section{Discussion}

In the present study, we have demonstrated that anticancer agent doxorubicin and ASO for miR221 could be successfully loaded on biologically derived EVs and efficiently delivered into HCC cells in culture, as well as into hepatic tumors when administered intravenously. Currently, a major problem facing conventional cancer therapy is effective drug delivery and drug targeting to the tumor site, unwanted injury to actively dividing healthy cells and side effects due to high toxicity. Biological nanoparticle-based drug delivery systems can resolve these problems to some extent. Since MNVs are presumably non-immune, non-toxic, and biocompatible with a suitable range of particle size $(80-150 \mathrm{~nm})$, they could enter the circulation and pass through cell membranes. These are important factors for optimum and effective drug delivery to the targeted tumor sites. The successful targeted delivery of anticancer agents into intrahepatic tumors could provide an effective and efficient modality for the treatment of HCC.
Secreted EVs and their contents reflect the physiological and pathological state of the cell [19]. It is assumed that the EVs serve as vehicles for intercellular communication and transfer of cytosolic proteins, lipids, mRNAs, and miRNAs. Exosomes have been successfully used for the target delivery of antitumor miRNA to breast cancer cells [20]. A number of nano-carriers based on different materials have been developed for the delivery of various anticancer agents. Among numerous nano-carriers including gold nanoparticles, ployethylene glycol-coated liposomes have been widely used [21]. However, liposomes have several drawbacks, including lack of efficiency of targeting and accelerated blood clearance [22, 23]. Therefore, it is important to develop a technique for efficient and targeted delivery of chemotherapeutic agents and ASO against upregulated miRNAs, which are relevant to cancer pathogenesis. In the current study, we effectively isolated nanovesicles of similar size from skim milk and characterized these using electron microscopy and NTA. Using a simple and easy technique, we effectively internalized anticancer agent doxorubicin and ASO for miRNA221, labeled the drug loaded MNVs with fluorescent lipophilic tracers, and successfully introduced into HCC cells in culture, as well as to intrahepatic tumors induced in immunocompromised mice. Since MNVs are biogenic and have phospholipid bilayers, they are biocompatible and can easily internalize chemotherapeutic agents. Taking advantage of tumor cell immune surveillance and easier access to the circulation in tumor cells compared to normal cells, drug loaded EVs could be taken up by tumor cells with minimal effects to normal cells.

We have observed a significant decrease in ZP of MNV after internalization of doxorubicin and clumping of doxMNV during NTA. These observations suggest an increased tendency of aggregation of doxorubicin-loaded MNV. However, we did not notice any significant decrease of ZP after internalization of anti-miR221 compared to plain $\mathrm{MNV}$. ZP is the potential difference between the dispersion medium and the stationary layer of fluid attached to the dispersed particle. If all the particles in suspension have a large negative or positive ZP they will tend to repel each other and there will be no tendency for the particles to come together. The mean $\mathrm{ZP}$ of plain $\mathrm{MNV}$ was $-33.1 \mathrm{mV}$, and very similar to that reported by Baddela et al. [24] ( $-29.5 \pm$ 6) for vesicles isolated from buffalo milk. Aggregation following internalization into HCC cells may happen with either dox-MNV or anti-miR221 MNV. Indeed, this has been observed in exosomes after internalization into tumor cells [25]. However, these do not appear to have a noticeable impact on cytotoxicity, or uptake within endosomes following uptake.

The current studies demonstrated that uptake of MNV by HCC cells in vitro is not affected by loading of doxorubicin. 

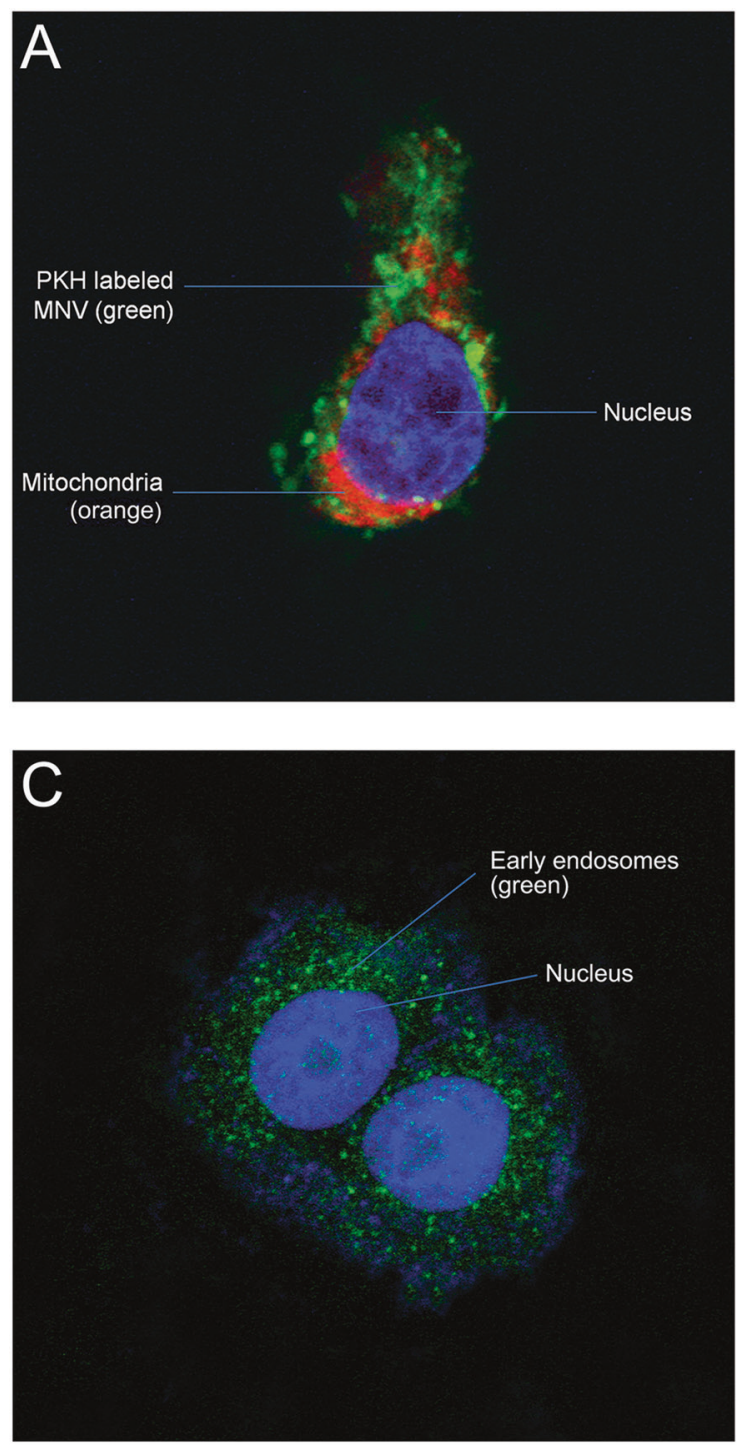

Fig. 7 Confocal Z-stack images of PLC/PRF/5 cells after internalization MNV and tracking of subcellular organelles. a MNV were labeled with PKH67 and the cells were treated with oxidized rosamine-based mitotracker probes. b MNV were labeled with DiI and the cells were incubated overnight with rabbit TGN46 antibody followed by second antibody conjugated with Alexa Flour 488. $\mathbf{c}$ PLC/PRF/5 cells cultured on chamber slides were fixed with formaldehyde, treated with rabbit EEA1 antibody overnight, washed, and incubated with anti-rabbit

Moreover, uptake occurs rapidly, with maximal uptake occurring within $4 \mathrm{~h}$. The rapid uptake supports the feasibility of delivery of anticancer agents, such as ASO to the liver using MNV. Thus, this approach has promise for the treatment of liver cancers, such as HCC. Furthermore, the presence of leaky blood vessels and the lack of proper lymphatic drainage in solid tumors could favor extravasation and retention of drug-loaded nanovesicles within tumors. In addition, uptake of drug-loaded MNV leads to nuclear fragmentation and cell death. Ahmed et al. [26] observed that exosomes-mediated delivery of intrinsic
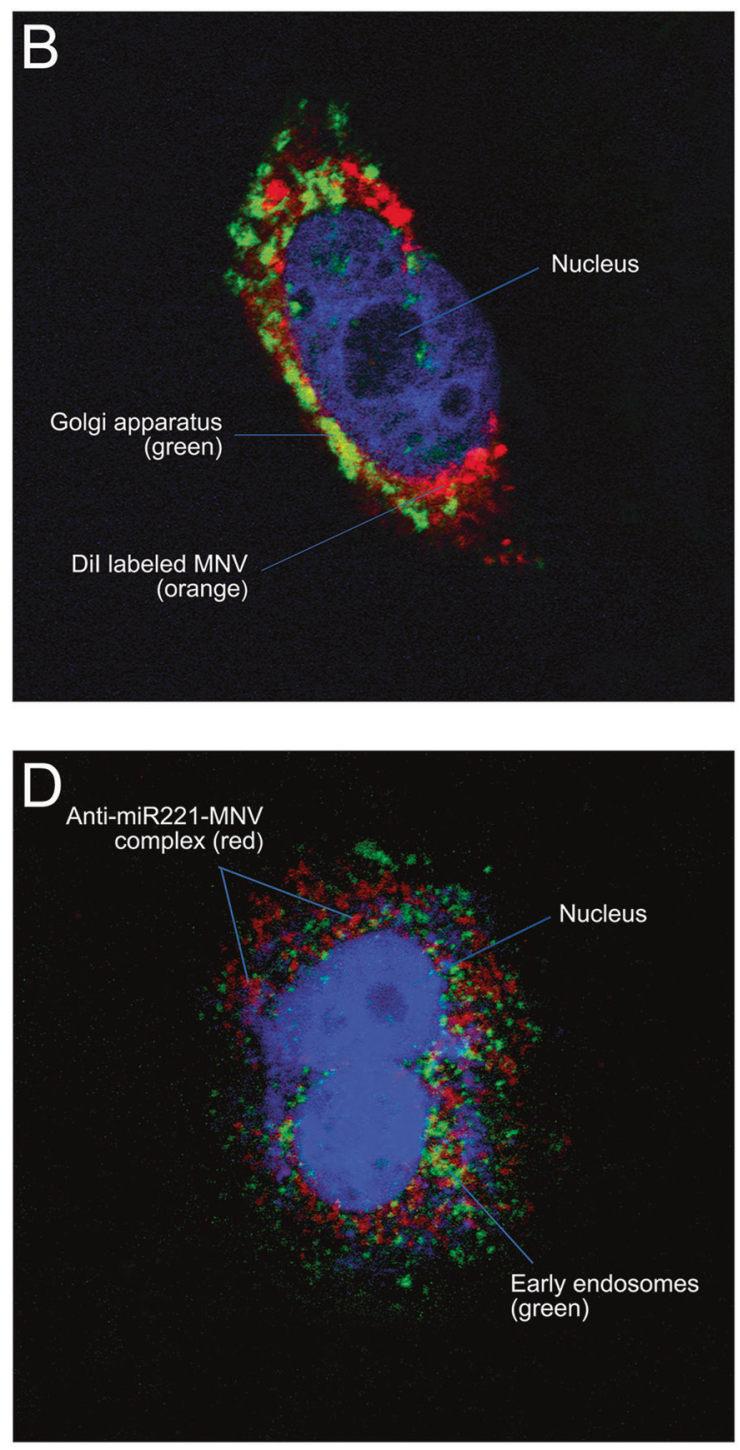

Alexa Flour 488. Perinuclear distribution of early endosomes. d MNV were internalized with biotinylated anti-miR221 oligonucleotides and treated with cells in culture for $24 \mathrm{~h}$. It was fixed and treated with rabbit EEA1 antibody overnight. The cells were then washed and simultaneously treated with anti-rabbit Alexa Flour 488 and streptavidin-conjugated Alexa Flour 647. Confocal Z-stack imaging did not show direct co-localization of loaded MNV with early endosomes

C-terminus domain of PTEN ablates proliferation, migration, and tumorigenesis in syngeneic breast tumor models. Thus, in addition to conventional anticancer agents, MNV could be used to deliver other constructs for experimental or therapeutic applications.

Biologically derived vesicles have many desirable features as a drug delivery system, such as long circulating half-life, intrinsic ability to target tissues, biocompatibility, and minimal or no inherent toxicity. Indeed, milk-derived exosomes did not exhibit cross-species reactivity and adverse immune and inflammatory responses [27]. The use 

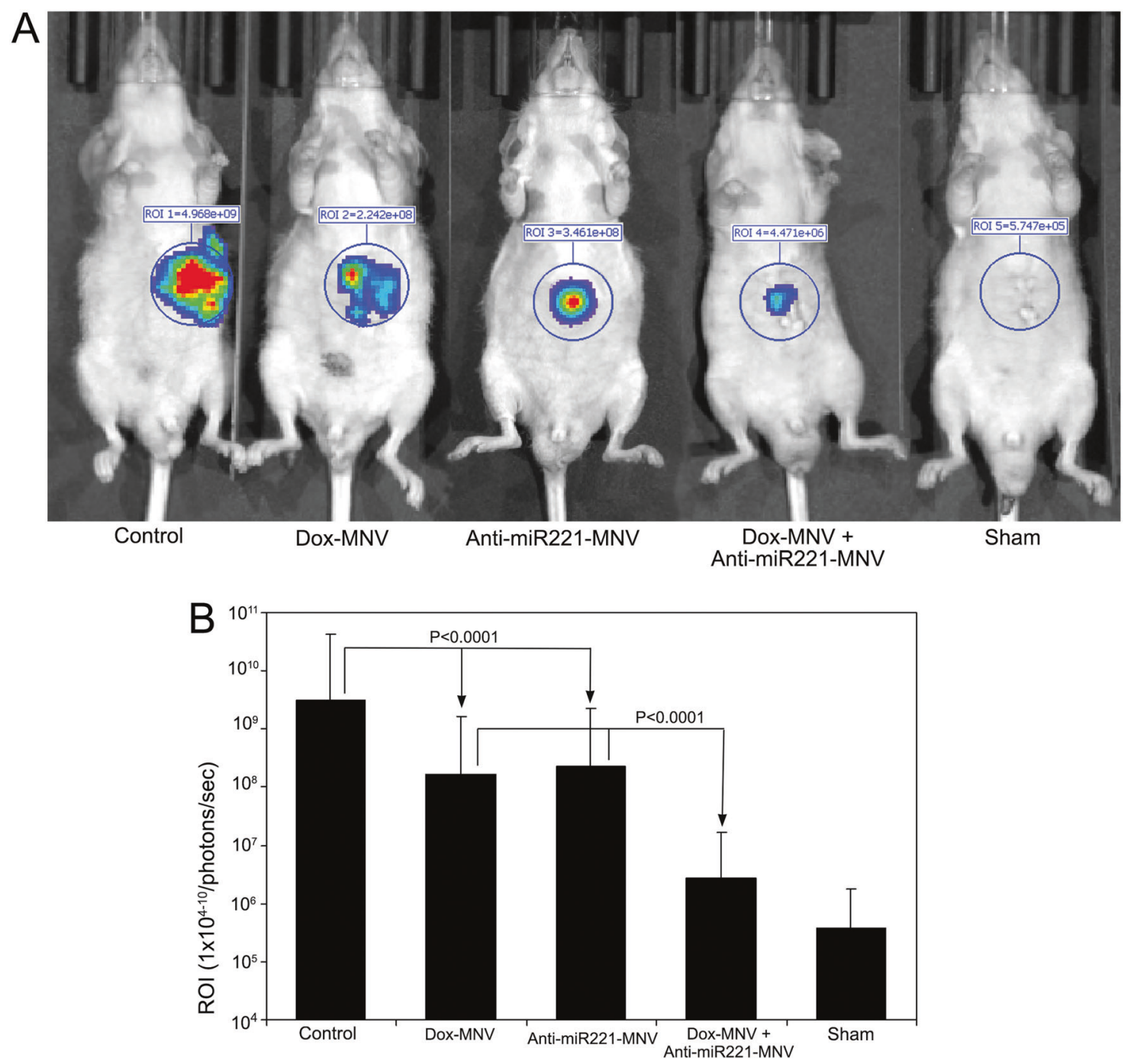

Fig. 8 Regression of intrahepatic tumors after the treatment with doxMNV. a Intrahepatic tumors were induced in immunocompromised mice after surgical implantation of PLC/PRF/5 HCC cells stably transfected with luciferase gene. Bioluminescence was monitored on every week using IVIS in vivo imaging system. When the signals exceeded $1 \times 10^{7}$ photons/s, the animals were injected through tail vein either with dox-MNV (400 $\mu$ g dox/kg body weight) or anti-miR221-

of EV-based drug delivery systems will provide an advantage over many issues that plague liposomal or polymeric drug delivery systems [28]. Some studies have reported a greater efficacy of EV-based therapies (e.g. for lung cancers), although we did not observe any differences in cytotoxicity in our cells and system [27]. We observed cell death with combinatorial therapies delivered via MNV uptake in a setting where there was no sign of toxicity in control cultures treated with plain MNV. Thus, our current study demonstrated that delivery of anticancer agents through MNV could be an efficient and effective method of drug delivery system for the treatment of various cancers.

Nanoparticle-mediated drug delivery is a very effective and potent method for efficient delivery and drug targeting
MNV (500 nmoles $/ \mathrm{kg}$ body weight) or both in combination or plain MNV thrice a week for 4 weeks. Treatment with dox-MNV and antimiR221-MNV resulted in marked decrease in tumor size and the amount of photons. b Quantitative representation of bioluminescence photons measured at the end of the study. The data are mean \pm SD of five animals in each group. $* P<0.001$, compared to respective plain MNV-treated control animals

$[29,30]$. Our current study showed that nanoparticle-based delivery of anticancer agents can be as effective as direct delivery of the same agents into HCC cells in culture. Besides, it was observed that anticancer agents delivered through MNV effectively inhibited cell division and induced death in all the three major HCC cell lines in culture. In addition, toxicity studies on multiple HCC cell lines indicated that the effect of treatment depends on the aggressiveness of the tumor cell phenotype, with PLC/PRF/ 5 being the most aggressive cell line and Hep3B being the least based on multiplication rate and cell death. Furthermore, results of our in vitro studies provided evidence that MNV could be effectively used for the delivery of anticancer agents into intrahepatic tumor cells. 
Information on mechanism of internalization and intracellular fate of nanovesicles are essential for optimal use as drug delivery vehicles. Live-cell microscopy studies have indicated that EVs are internalized through endocytosis pathway, trapped in vesicles, and transported to perinuclear region [31]. Fluorescence microscopy showed a very diffused and non-compartmentalized subcellular localization of polycaprolactone/maltodextrin nanocarriers with possible intranuclear localization and minor co-localization in the lysosomes in prostrate cancer cell line [32]. Endosomes and mitochondria have been implicated in the uptake of TGN peptide-modified nanoparticles by glioma cells [33]. However, our studies do not indicate a definite role for endosomal uptake of MNV or interaction with either mitochondria or Golgi apparatus. The possibility of association of MNVs following their uptake with early endosomes needs to be fully clarified.

Previously we have demonstrated that hepatic orthotopic tumors treated with chol-anti-miR221 decreased tumor cell proliferation, increased apoptosis, and cell-cycle arrest with a significant increase of mouse survival period [7]. It was also shown that exosomes derived from miR122-modified adipose tissue-derived mesenchymal stem cells increase chemosensitivity in HCC cells [34]. We showed that MNVmediated delivery of doxorubicin through intravenous administration markedly decreased experimentally induced hepatic tumors in nude mice within 4 weeks of treatment. The therapy also prevented metastasis of tumor cells to other organs. Thus, the study provided evidence that MNV could be successfully used for the target delivery of chemotherapeutic agents and ASO for tumor-specific miRNAs into intrahepatic tumors, and would be an effective modality for treatment of HCC.

In conclusion, this study demonstrates that biologically derived vesicles can be isolated from skim milk, and effectively used for the delivery of therapeutic agents, such as anticancer drugs or ASO into tumor cells in vitro and in vivo. The study thereby emphasizes the potential role of MNV as a drug-delivery platform for the treatment of primary human liver tumors.

Acknowledgements The authors are thankful to Ms. Sarah Nix for her excellent technical assistance with the animal experiments.

Funding This work was supported in part by the Grant UH3 TR000884 from the Office of the Director, National Institutes of Health and National Center for Advancing Translational Sciences (NCATS) to Tushar Patel.

\section{Compliance with ethical standards}

Conflict of interest The authors declare that they have no conflict of interest.

\section{References}

1. Chun JM, Kwon HJ, Sohn J, et al. Prognostic factors after early recurrence in patients who underwent curative resection for hepatocellular carcinoma. J Surg Oncol. 2011;103:148-51.

2. Torre LA, Bray F, Siegel RL, et al. Global cancer statistics, 2012. CA Cancer J Clin. 2015;65:87-108.

3. Thorgeirsson SS, Grisham JW. Molecular pathogenesis of human hepatocellular carcinoma. Nat Genet. 2002;31:339-46.

4. George J, Patel T. Noncoding RNA as therapeutic targets for hepatocellular carcinoma. Semin Liver Dis. 2015;35:63-74.

5. Di Leva G, Garofalo M, Croce CM. MicroRNAs in cancer. Annu Rev Pathol. 2014;9:287-314.

6. Ha M, Kim VN. Regulation of microRNA biogenesis. Nat Rev Mol Cell Biol. 2014;15:509-24.

7. Park JK, Kogure T, Nuovo GJ, et al. miR-221 silencing blocks hepatocellular carcinoma and promotes survival. Cancer Res. 2011;71:7608-16.

8. Dubbelboer IR, Lilienberg E, Ahnfelt E, et al. Treatment of intermediate stage hepatocellular carcinoma: a review of intrahepatic doxorubicin drug-delivery systems. Ther Deliv. 2014;5:447-66.

9. Yang G, Jiang O, Ling D, et al. MicroRNA-522 reverses drug resistance of doxorubicin-induced HT29 colon cancer cell by targeting ABCB5. Mol Med Rep. 2015;12:3930-6.

10. Qu J, Zhao L, Zhang P, et al. MicroRNA-195 chemosensitizes colon cancer cells to the chemotherapeutic drug doxorubicin by targeting the first binding site of BCL2L2 mRNA. J Cell Physiol. 2015;230:535-45.

11. Davis ME, Chen ZG, Shin DM. Nanoparticle therapeutics: an emerging treatment modality for cancer. Nat Rev Drug Discov. 2008;7:771-82.

12. Johnsen KB, Gudbergsson JM, Skov MN, et al. A comprehensive overview of exosomes as drug delivery vehicles - endogenous nanocarriers for targeted cancer therapy. Biochim Biophys Acta. 2014;1846:75-87.

13. Vlassov AV, Magdaleno S, Setterquist R, Conrad R. Exosomes: current knowledge of their composition, biological functions, and diagnostic and therapeutic potentials. Biochim Biophys Acta. 2012;1820:940-8.

14. Lee Y, El Andaloussi S, Wood MJ. Exosomes and microvesicles: extracellular vesicles for genetic information transfer and gene therapy. Hum Mol Genet. 2012;21:R125-34.

15. El Andaloussi S, Lakhal S, Mager I, Wood MJ. Exosomes for targeted siRNA delivery across biological barriers. Adv Drug Deliv Rev. 2013;65:391-7.

16. Valadi H, Ekstrom K, Bossios A, et al. Exosome-mediated transfer of mRNAs and microRNAs is a novel mechanism of genetic exchange between cells. Nat Cell Biol. 2007;9:654-9.

17. Ettelaie C, Collier ME, Maraveyas A, Ettelaie R. Characterization of physical properties of tissue factor-containing microvesicles and a comparison of ultracentrifuge-based recovery procedures. $\mathrm{J}$ Extracell Vesicles 2014;3:23592.

18. Haga H, Yan IK, Takahashi K, et al. Tumour cell-derived extracellular vesicles interact with mesenchymal stem cells to modulate the microenvironment and enhance cholangiocarcinoma growth. J Extracell Vesicles. 2015;4:24900.

19. Barger JF, Rahman MA, Jackson D, Acunzo M, Nana-Sinkam SP. Extracellular miRNAs as biomarkers in cancer. Food Chem Toxicol. 2016;98:66-72.

20. Ohno S, Takanashi M, Sudo K, et al. Systemically injected exosomes targeted to EGFR deliver antitumor microRNA to breast cancer cells. Mol Ther. 2013;21:185-91. 
21. Mitchell MJ, Chen CS, Ponmudi V, Hughes AD, King MR. Eselectin liposomal and nanotube-targeted delivery of doxorubicin to circulating tumor cells. J Control Release. 2012;160:609-17.

22. Ishida T, Kiwada H. Accelerated blood clearance (ABC) phenomenon upon repeated injection of PEGylated liposomes. Int $\mathbf{J}$ Pharm. 2008;354:56-62.

23. Abu Lila AS, Uehara Y, Ishida T, Kiwada H. Application of polyglycerol coating to plasmid DNA lipoplex for the evasion of the accelerated blood clearance phenomenon in nucleic acid delivery. J Pharm Sci. 2014;103:557-66.

24. Baddela VS, Nayan V, Rani P, Onteru SK, Singh D. Physicochemical biomolecular insights into buffalo milk-derived nanovesicles. Appl Biochem Biotechnol. 2016;178:544-57.

25. Suetsugu A, Honma K, Saji S, et al. Imaging exosome transfer from breast cancer cells to stroma at metastatic sites in orthotopic nude-mouse models. Adv Drug Deliv Rev. 2013;65:383-90.

26. Ahmed SF, Das N, Sarkar M, et al. Exosome-mediated delivery of the intrinsic C-terminus domain of PTEN protects it from proteasomal degradation and ablates tumorigenesis. Mol Ther. 2015;23:255-69.

27. Munagala R, Aqil F, Jeyabalan J, Gupta RC. Bovine milk-derived exosomes for drug delivery. Cancer Lett. 2016;371:48-61.
28. Ha D, Yang N, Nadithe V. Exosomes as therapeutic drug carriers and delivery vehicles across biological membranes: current perspectives and future challenges. Acta Pharm Sin B. 2016;6:287-96.

29. Bae YH, Park K. Targeted drug delivery to tumors: myths, reality and possibility. J Control Release. 2011;153:198-205.

30. Mishra N, Yadav NP, Rai VK, et al. Efficient hepatic delivery of drugs: novel strategies and their significance. Biomed Res Int. 2013;2013:382184.

31. Tian T, Wang Y, Wang H, Zhu Z, Xiao Z. Visualizing of the cellular uptake and intracellular trafficking of exosomes by livecell microscopy. J Cell Biochem. 2010;111:488-96.

32. Korang-Yeboah M, Gorantla Y, Paulos SA, et al. Polycaprolactone/maltodextrin nanocarrier for intracellular drug delivery: formulation, uptake mechanism, internalization kinetics, and subcellular localization. Int J Nanomed. 2015;10:4763-81.

33. Gao H, Yang Z, Zhang S, Pang Z, Jiang X. Internalization and subcellular fate of aptamer and peptide dual-functioned nanoparticles. J Drug Target. 2014;22:450-9.

34. Lou G, Song X, Yang F, et al. Exosomes derived from miR-122-modified adipose tissue-derived MSCs increase chemosensitivity of hepatocellular carcinoma. J Hematol Oncol. $2015 ; 8: 122$. 